\title{
Alport syndrome
}

\author{
Karki $\mathrm{P}^{1}$, Shrestha $\mathrm{JK}^{2}$ \\ ${ }^{1}$ Biratnagar Eye Hospital, Morang, ${ }^{2} \mathrm{BP}$ Koirala Lion's Center for Ophthalmic Studies \\ Institute of Medicine, Kathmandu
}

\begin{abstract}
Alport syndrome is an oculo-renal syndrome characterized by a triad of clinical findings consisting of hemorrhagic nephritis, sensorineural hearing loss and characteristic ocular findings. We report a young male patient who presented with painless diminution of vision associated with hearing loss. The importance of ophthalmic evaluation for suspecting the disease is highlighted.
\end{abstract}

Keywords: Alport syndrome, sensorineural hearing loss, micro-hematuria, oculo-renal syndrome

\section{Background}

Alport syndrome is an oculo-renal syndrome characterized by a triad of clinical findings consisting of hemorrhagic nephritis with sensorineural hearing loss and characteristic ocular findings. It was first reported by Cecil Alport in 1927. The basic defect in Alport's syndrome is abnormal basement membrane type IV collagen. The gene involved, COL4A5, is located in the q22 region of the $\mathrm{X}$ chromosome (Atkin et al 1988 \& Barker et al 1990). It is a dominantly inherited Xlinked disorder with an approximate incidence of 1 in 5000 persons (Atkin et al 1988).

\section{Case}

A 24-year-old male from western Nepal presented with bilateral progressive painless diminution of vision and hearing loss for the last 8 years. There was a history of several visits to other eye hospitals and clinics in the past where only glasses had been prescribed, the result of which was a little relief. The patient did not have any other systemic complaints. There was also no positive family history of similar complaints.

The patient was seen in the out-patient department of ophthalmology where detailed ocular examination revealed bilateral visual impairment with best-corrected

Received: 30.12 .2008 Accepted: 25.06.2009

Correspondence and reprint request to: Dr Pratap Karki, MD

Biratnagar Eye Hospital

Morang, Nepal

E-mail: pkarki10@yahoo.co.in distance visual acuity of 6/36 and near vision of $\mathrm{N} 10$. Refraction showed compound myopic astigmatism with OD $-1.0 \mathrm{D} x-0.5 \mathrm{D}$ at $180^{\circ}$ and OS $-0.75 \mathrm{D} \times-0.25 \mathrm{D}$ at $180^{\circ}$. Anterior segment evaluation under slit-lamp revealed bilateral clear cornea and normal anterior chamber with bilateral anterior lenticonus with anterior sub-capsular cataract [Figure 1\&2]. On distant direct ophthalmoscopy, bilateral oil droplet appearance was seen. Fundus evaluation also revealed bilateral pigmentary changes in the macula. Blood pressure was found to be $140 / 100 \mathrm{mmHg}$. Systemic evaluation showed normal central nervous, respiratory and cardiovascular systems. Bilateral severe sensorineural hearing loss was diagnosed on audiometry. Upon suspicion of oculo-renal syndrome, a routine urine examination revealed protenuria $3+$ with hematuria and trace sugar.

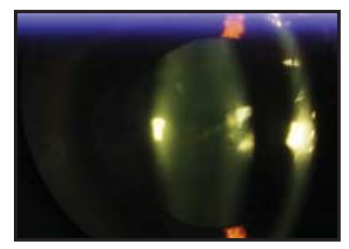

Right Eye (Figure 1): anterior lenticonus with anterior subcapsular cataract

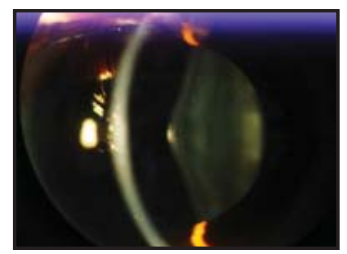

Left Eye (Figure 2): anterior lenticonus with anterior subcapsular cataract 
Based on the above findings, the diagnosis of Alport's syndrome was made and the patient was referred to a nephrologists for confirmation of the diagnosis. Further investigation revealed a raised urea $(12.9 \mathrm{mmol} / \mathrm{L})$, with a raised creatinine (587 micro mol/l) and 24-hour-urine protein of $4.2 \mathrm{~g} /$ day. The patient was finally commenced on enalapril $5 \mathrm{mg} /$ day in view of controlling proteinuria and was asked to be followed up in a month.

\section{Discussion}

The diagnosis of Alport's syndrome consists of

1. family history of hematuria, progressing mostly in males to end-stage renal disease (ESRD),

2. thickening and splitting of the glomerular basement membrane detected by electron microscopy,

3. progressive, high-frequency, sensorineural deafness and

4. anterior lenticonus and perimacular flecks.

The presence of three out of the above four proposed diagnostic criteria establishes the diagnosis of Alport syndrome. However, all criteria need not be present to make a clinical diagnosis. Bilateral sensorineural hearing deafness with persistent micro-hematuria is highly suggestive of Alport syndrome even in the absence of relevant family history (Sessa et al 2003). No positive family history was known in our case.

The male sex is associated with an earlier onset and a more severe progression of renal disease in Alport syndrome. Females on the other hand have a variable course with only a few severely affected by the disease. In an average age of 25 years, $94 \%$ males and $3 \%$ females present with renal insufficiency with elevated serum creatinine (Sessa et al 2003). The male sex in our case is hence associated with a poor prognosis as shown by the results of renal function tests.

Our experience with the above case emphasizes the role of ophthalmologists in the diagnosis of Alport syndrome. Without the findings revealed during the ocular examination, the diagnosis would probably have been missed or delayed.

\section{References}

Alport AC (1927). Hereditary familial congenital haemorrhagic nephritis. Br Med J; 1:504

Atkin CL, Gregory MC, Border WA (1988). Alport syndrome. In: Schrier RW (ed): Diseases of the Kidney. Boston, Little Brown: 617

Atkin CL, Hasstedt SL, Menlove L et al (1988). Mapping of Alport syndrome to the long arm of the X chromosome. Am J Hum Genet; 42:249

Barker DF, Hostikka SL, Zhou J (1990). Identification of mutations in the COL4A5 collagen gene in Alport syndrome. Science; 248:1224

Sessa A, Meroni M (2003). Alport syndrome. Orphanet encyclopedia.

Source of support: nil. Conflict of interest: none 